\title{
Thoracic Wall Metastasis from an Occult Thyroid Follicular Carcinoma
}

\author{
${ }^{1} \mathrm{NJ}$ Nawarathna, ${ }^{2} \mathrm{NR}$ Kumarasinghe, ${ }^{3} \mathrm{D}$ Chandrasekara, ${ }^{4} \mathrm{P}$ Rathnayake, ${ }^{5} \mathrm{BMRS}$ Balasooriya, ${ }^{6} \mathrm{RJK}$ Senevirathne
}

\begin{abstract}
Occult thyroid carcinoma presenting with clinically apparent metastasis is rare and is a diagnostic challenge. Here, we report a 67-year-old male who presented with a left side chest wall mass resembling a soft tissue sarcoma of 1 year duration. The mass showed rapid enlargement at the latter end of its course, following an initial asymptomatic period. Imaging studies showed a soft tissue mass eroding into several ribs. Wide local excision with primary reconstruction was performed. Histological studies and immune staining revealed metastasis from a follicular thyroid carcinoma. Total thyroidectomy followed, confirming the diagnosis. Postoperatively, radioisotope ablation $\left({ }^{131}\right)$ was done. A suppression dose of thyroxin was continued with regular thyroglobulin assays. Painful bone metastasis responded well to analgesics, bisphosphonates and external beam radiotherapy. Follicular carcinoma comprises 10 to $15 \%$ of thyroid malignancies. Localized thyroid carcinoma has a very good prognosis, 10 years survival rates reducing by $50 \%$ with metastatic disease. Commonly, thyroid cancer presents as detectable thyroid nodules, $25 \%$ having metastasis. In contrast, metastatic manifestations are reported in less than $5 \%$ of occult thyroid cancers.
\end{abstract}

Keywords: Follicular carcinoma, Occult thyroid carcinoma, Thoracic wall metastasis.

How to cite this article: Nawarathna NJ, Kumarasinghe NR, Chandrasekara D, Rathnayake P, Balasooriya BMRS, Senevirathne RJK. Thoracic Wall Metastasis from an Occult Thyroid Follicular Carcinoma. World J Endoc Surg 2014;6(3):119-122.

Source of support: Nil

Conflict of interest: None

\section{INTRODUCTION}

Manifestation of secondary deposits from a silent thyroid cancer is one presentation of the condition defined as occult thyroid carcinoma. ${ }^{1}$ Approximately, $25 \%$ of metastatic $^{2}$ spread from differentiated thyroid cancer (DTC)

\footnotetext{
${ }^{1,3}$ Registrar, ${ }^{2}$ Senior Registrar

${ }^{4,6}$ Consultant, ${ }^{5}$ Research Assistant

${ }^{1-3}$ Department of Surgery, Teaching Hospital, Kandy, Sri Lanka

${ }^{4}$ Department of Pathology, Teaching Hospital, Kandy, Sri Lanka

${ }^{5}$ Faculty of Medicine, University of Peradeniya, Kandy, Sri Lanka

${ }^{6}$ Department of General Surgery, Teaching Hospital, Kandy Sri Lanka
}

Corresponding Author: NJ Nawarathna, Registrar, Department of Surgery, Teaching Hospital, Kandy, Sri Lanka, Phone: 094718130181, e-mail: nadeeshanawarathna@yahoo.com is to bone. Secondary deposit from occult thyroid cancer is rare ${ }^{3}$ and presents a challenge to the clinician in its diagnosis. The presence of distant metastasis is reported to decrease 10 years survival rate by $50 \%{ }^{4}$ In this paper, we present a middle aged male who presented with a thoracic wall mass suggestive of a soft tissue tumor. Histological analysis revealed a metastatic deposit of an occult follicular thyroid cancer.

\section{CASE REPORT}

A 67-year-old male, presented with a lump on the left side of his chest for duration of 8 months. Initial gradual enlargement was noted with rapid enlargement over the preceding 2 months associated with intermittent pain. He was a known diabetic on oral hypoglycemics.

Clinical examination revealed a painless mass of $10 \times$ $15 \mathrm{~cm}$ on the left side posterolateral chest. Further examination suggested attachment to the thoracic wall (Fig. 1). Regional lymphadenopathy was not present, and organ system evaluation was unremarkable. The provisional diagnosis was soft tissue sarcoma.

Contrast-enhanced computerized tomography scan revealed a mixed density mass (cystic and solid areas) within the chest wall eroding into the 9th, 10th and 11th ribs (Fig. 2). Its deep surface was seen to protrude into the pleural space, yet the pleura was intact. Mediastinal node enlargement as well as pulmonary or liver secondaries was not seen. Fine needle aspiration of the mass done twice was inconclusive and revealed blood only.

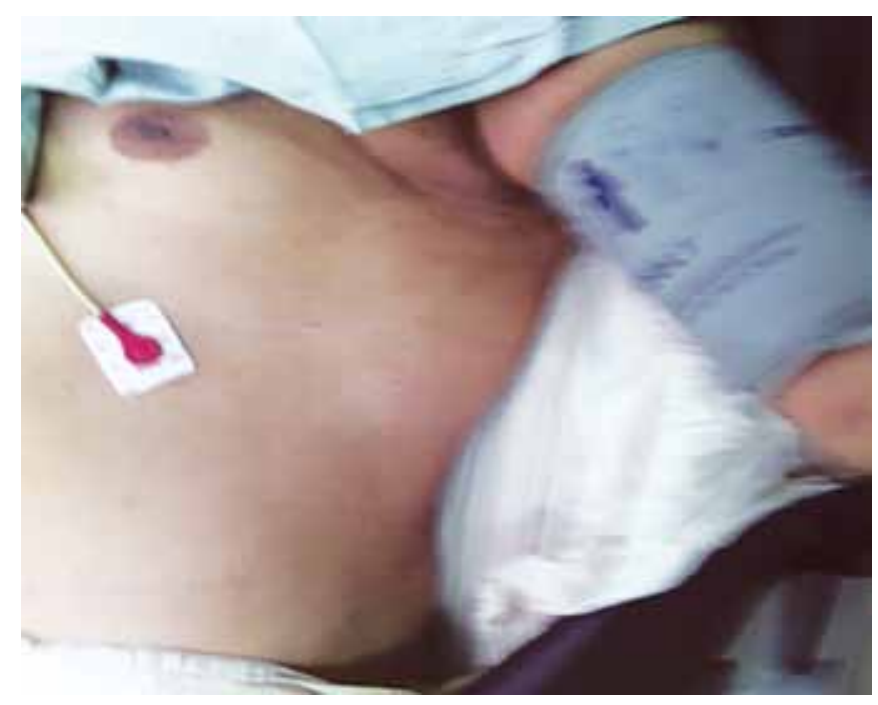

Fig. 1: Thoracic wall metastasis 
A left sided thoracotomy was performed and the mass surgically excised with resection of the involved ribs. The thoracic wall defect was primarily repaired with a prosthetic mesh and primary closure was achieved.

Macroscopically, it an encapsulated mass with solid and cystic areas was seen. Hematoxylin and eosin stain showed packed small follicles with empty lumina. The tumor infiltrated the capsule and skeletal muscle (Fig. 3). Appearances were highly suggestive of a metastatic follicular carcinoma of the thyroid. Malignant neoplasms of renal and pulmonary origin were other differential diagnoses which were excluded by immunohistochemistry (Figs 4 to 6).
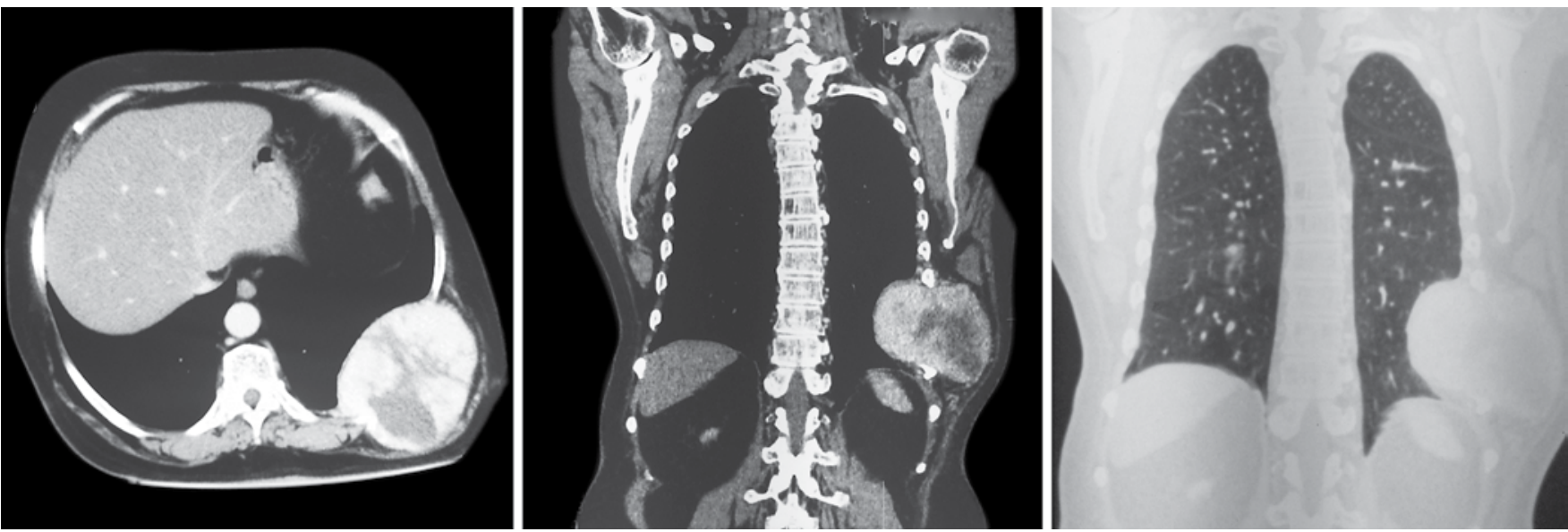

Fig. 2: CT film of thoracic wall metastasis

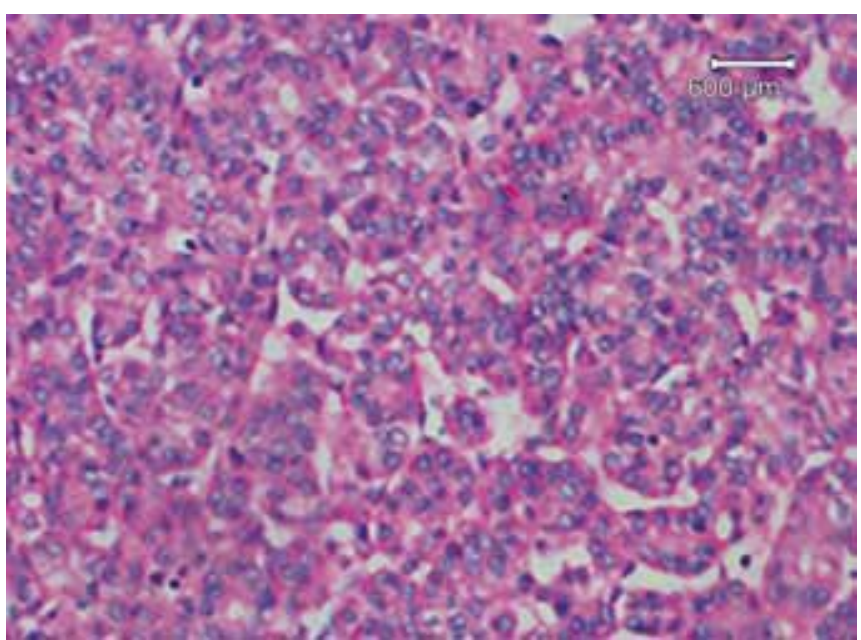

Fig. 3: High power view of thoracic wall metastasis from the follicular carcinoma of the thyroid

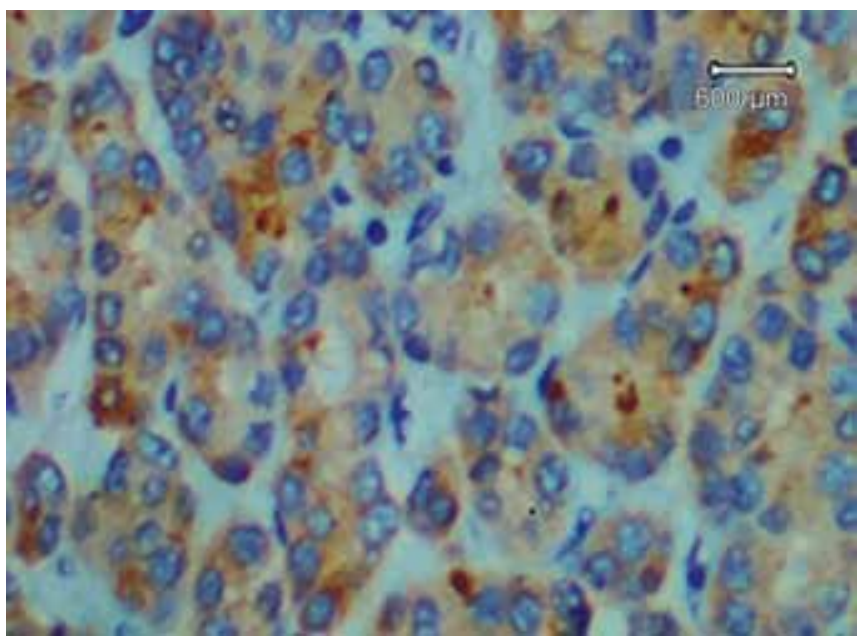

Fig. 5: Metastasis was immunonegative for epithelial membrane antigen

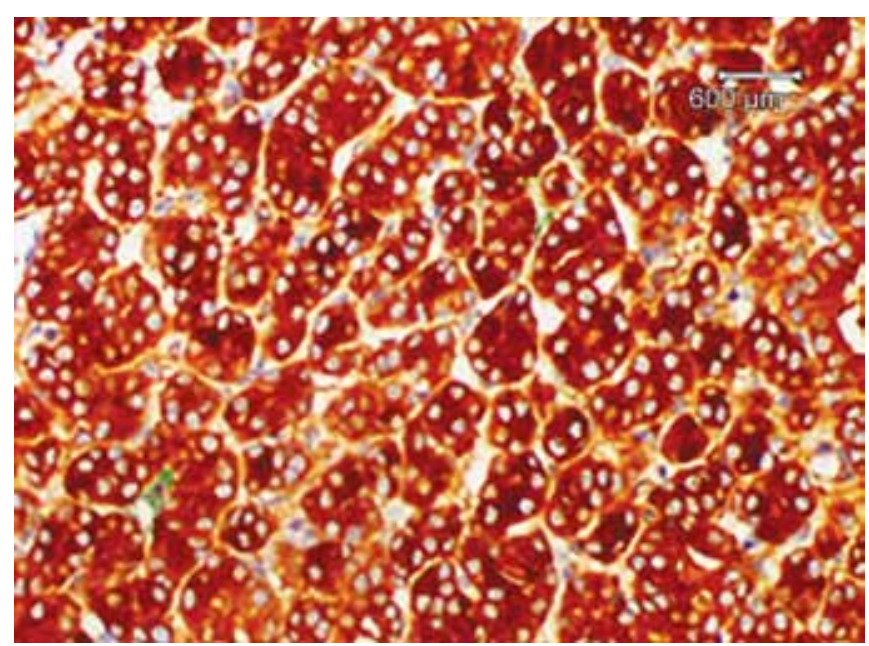

Fig. 4: Metastasis was immunopositive for thyroglobulin

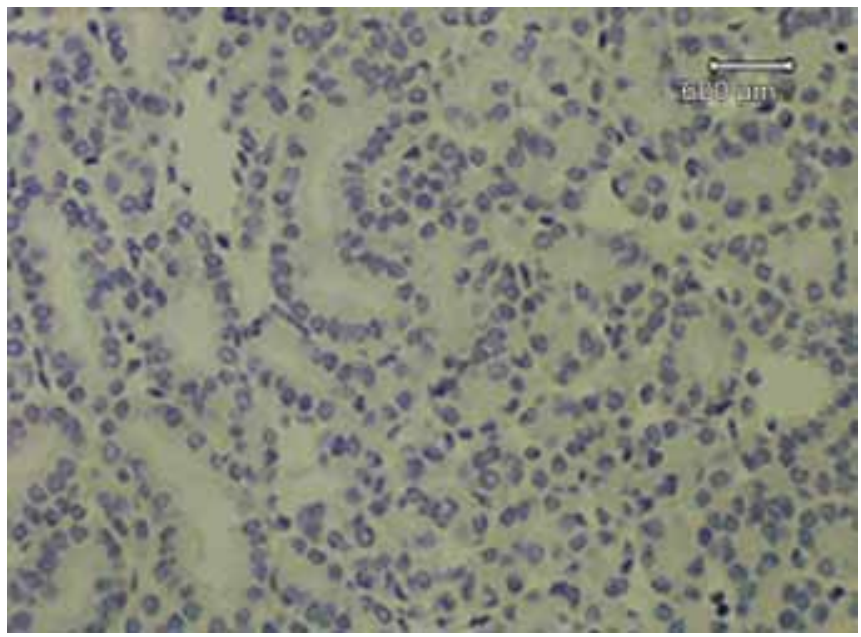

Fig. 6: Metastasis was immunonegative for carcinoembryonic antigen 


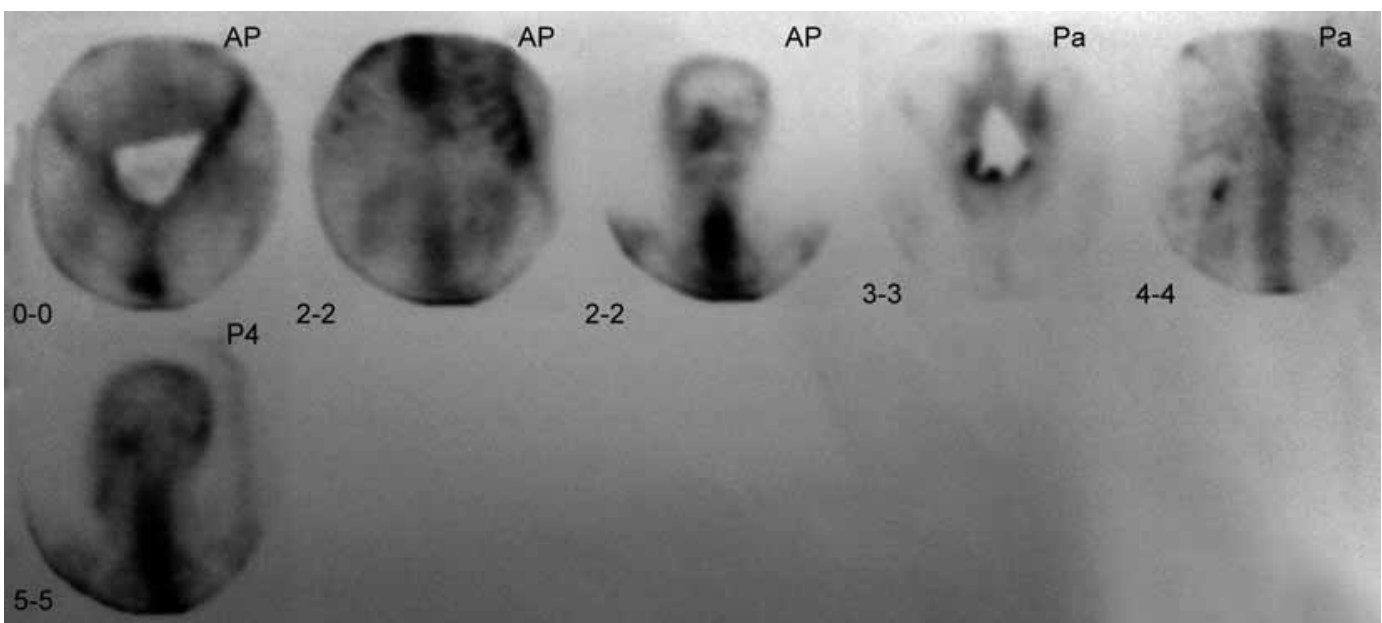

Fig. 7: Tc-99m methylene diphosphonate bone scan; high uptake areas are suggestive of bone metastasis

Further radiological imaging was performed on the thyroid and abdomen. Imaging of the thyroid gland revealed a multinodular goiter with a suspicious nodule of the right lobe. Abdominal imaging studies were normal. Radioisotope scanning with Tc-99m methylene diphosphonate showed significant tracer uptake of multiple ribs on both sides, compatible with bone metastasis (Fig 7).

Patient underwent total thyroidectomy. Histology confirmed a follicular carcinoma of the thyroid. Suppression doses of thyroxin were prescribed in the postoperative period followed by $\mathrm{I}^{131}$ ablation. Pain from metastatic bone lesions were managed with analgesics, bisphosphonates and physiotherapy.

\section{DISCUSSION}

Differentiated thyroid carcinoma is the commonest endocrine malignancy. ${ }^{5}$ Papillary carcinoma comprises the majority whilst $10 \%$ are follicular carcinomas. The overall survival from localized thyroid carcinoma is 85 to $90 \%$ at 10 years but reduces by $50 \%$ in metastatic disease. About $25 \%$ of follicular carcinomas eventually develop metastasis, with increasing age being a significant risk factor.

Reports of metastatic disease as the initial presentation of occult thyroid follicular carcinoma are limited, with an incidence of about 3 to $4 \%$ according to previous studies. $^{7,8}$

Occult thyroid carcinoma can be categorized as; incidentally found thyroid carcinoma or microcarcinoma in thyroidectomised specimens for benign disease or at autopsy, incidentally detected papillary thyroid microcarcinoma on imaging studies, clinically apparent metastasis from a clinically undetectable thyroid malignancy and thyroid cancer localized in ectopic thyroid tissue. ${ }^{1}$

In this case, our patient presented with clinically apparent metastatic deposits. Skeletal metastases of carcinoma of the thyroid normally present with pain but maybe clinically silent. Pain maybe due to release of cytokines from the tumor or maybe due to pressure or mass effect within the bone. Additionally, patients may present with fractures in some situations of skeletal metastasis.

The main mode for spread of follicular thyroid carcinoma is the hematogenous route. The commonest site of metastasis being, the lung followed by bone. ${ }^{9,10}$ More than $80 \%$ of bone metastasis is to the axial skeleton, commonly the vertebrae, ribs and pelvis. The excised soft tissue mass in this patient was well encapsulated and attached to ribs.

Hematoxylin and eosin staining of the excised tumor showed cells arranged in small follicles, which were empty. Thyroid follicular carcinoma could not be confirmed by light microscopy. Differential diagnoses included renal and lung malignancy. A positive thyroglobulin and epithelial membrane antigen (EMA) as well as negative carcinoembryonic antigen (CEA) in immunohistochemistry studies confirmed the origin of the tumor.

Ultrasonic imaging of the neck showed a multinodular goiter with a suspicious nodule on its right side. Lung and renal malignancies were excluded. The patient underwent total thyroidectomy and the histology confirmed a follicular carcinoma of the thyroid gland. Postoperatively, he was started on suppression thyroxin doses. Subsequently, a methylene diphosphonate Tc-99m bone scan was performed as a staging investigation. ${ }^{6}$ Multiple hot areas of the axial skeleton were compatible with bone metastasis from a thyroid follicular carcinoma.

Surgery is the main form of treatment for resectable metastatic differentiated thyroid carcinoma, ${ }^{11,12}$ followed by $\mathrm{I}^{131}$ ablation. Since our patient had multiple painful bone deposits we started him on analgesics and bisphosphonates $^{13}$ to which he responded. Skeletal metastasis have osteolytic effects on bone thus the anti osteoclastic activity of bisphosphonates are known to have a beneficial effect on such patients. ${ }^{11}$ However when surgery is an impractical option, other modalities need to be considered. External beam radiotherapy ${ }^{12}$ is an alternative for iodine unresponsive metastatic lesions. 


\section{REFERENCES}

1. Boucek J, Kastner J, Skrivan J, Grosso E, Gibelli B, et al. Occult thyroid carcinoma. Acta Otorhinolaryngol Ital 2009 Dec;29(6): 296-304.

2. Kelessis NG, Prassas EP, Dascalopoulou DV, Apostolikas NA, Tavemaraki AP, et al. Unusual metastatic spread of follicular thyroid carcinoma: report of a case. Surg Today 2005;35(4): 300-303.

3. Sevinc A, Buyukberber S, Sari R, Baysal T, Mizrak B. Follicular thyroid cancer presenting initially with soft tissue metastasis. JPN J Clin Oncol 2000 Jan;30(1):27-29.

4. Cooper DS, Doherty GM, Haugen BR, Kloos RT, Lee SL, Mandel SJ, Mazzaferri EL, McIver B, Pacini F, Schlumberger $\mathrm{M}$, et al. Revised American thyroid association management guidelines for patients with thyroid nodules and differentiated thyroid cancer. Thyroid 2009 Nov;19(11):1167-1214.

5. Ozdemri N, Senoglu M, Acar UD, et al. Skull metastasis of follicular thyroid carcinoma. Acta Neurochirurgica 2004 Oct; 146(10):1155-1158.

6. Emerick GT, Duh QY, Siperstein AE, Burrow GN, Clark OH. Diagnosis, treatment and outcome of follicular thyroid carcinoma. Cancer 1993 Dec;72(11):3287-3295.
7. Shaha AR, Shah JP, Loree TR. Differentiated thyroid cancer presenting initially with distant metastasis. Am J Surg 1997 Nov;174(5):474-476.

8. Sevinc A, Buyukberber S, Sari R, Baysal T, Mizrak B. Follicular thyroid cancer presenting initially with soft tissue metastasis JPN J Clin Oncol 2000 Jan;30(1):27-29.

9. Parlea L, Fahim L, Munoz D, et al. Follicular carcinoma of the thyroid with aggressive metastatic behavior in a pregnant woman: report of a case and review of the literature. Hormones 2006;5(4):295-302.

10. Wexler JA. Approach to the thyroid cancer patient with bone metastases. J Clin Endocrinol Metab 2011 Aug;96(8):2296-2307.

11. Orita $Y$, Sugitani I, Matsuura M, et al. Prognostic factors and the therapeutic strategy for patients with bone metastasis from differentiated thyroid carcinoma. Surgery 2010 Mar; 147(3):424-431.

12. Lutz S, Berk L, Chang E, et al. Palliative radiotherapy for bone metastases: an ASTRO evidence based guideline. Int J Radiat Oncol Biol Phys 2011 Mar;79(4):965-976.

13. Henry DH, Costa L, Goldwasser F, et al. Randomized, double blind study of denosumab versus zoledronic acid in the treatment of bone metastases in patients with advanced cancer (excluding breast and prostate cancer) or multiple myeloma. J Clin Oncol 2011 Mar 20;29(9):1125-1132. 